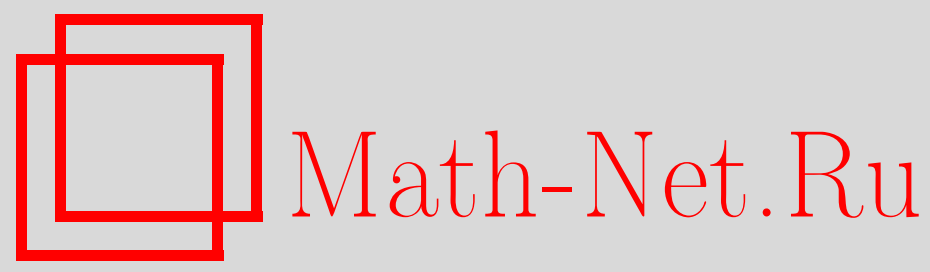

К. Б. Сабитов, С. Л. Бибакова, Построение собственных функций задачи Трикоми-Неймана для уравнения смешанного типа с характеристическим вырождением и их применение, Матем. заметки, 2003, том 74, выпуск 1, 76-87

DOI: https://doi.org/10.4213/mzm247

Использование Общероссийского математического портала Math-Net.Ru подразумевает, что вы прочитали и согласны с пользовательским соглашением http://www. mathnet.ru/rus/agreement

Параметры загрузки:

IP : 52.90 .164 .192

26 апреля 2023 г., 14:13:32

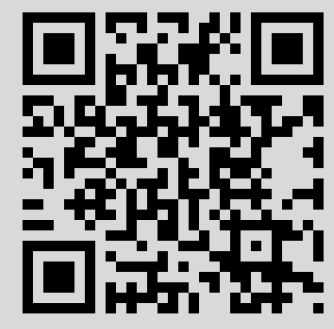




\section{ПОСТРОЕНИЕ СОБСТВЕННЫХ ФУНКЦИЙ ЗАДАЧИ ТРИКОМИ-НЕЙМАНА ДЛЯ УРАВНЕНИЯ \\ СМЕШАННОГО ТИПА С ХАРАКТЕРИСТИЧЕСКИМ \\ ВЫРОЖДЕНИЕМ И ИХ ПРИМЕНЕНИЕ}

К. Б. Сабитов, С. Л. Бибакова

Для уравнения смешанного типа

$$
L_{\alpha} u \equiv u_{x x}+y u_{y y}+\alpha u_{y}+\lambda u=0,
$$

где $0<\alpha<1, \lambda$ - комплексный параметр, в специальной области методом разделения переменных найдены собственные значения и построена система соответствующих собственных функций спектральной задачи Трикоми-Неймана. Построено решение задачи Трикоми-Неймана в виде суммы биортогонального ряда.

Библиография: 14 названий.

1. Постановка задачи. Рассмотрим уравнение

$$
L_{\alpha} u \equiv u_{x x}+y u_{y y}+\alpha u_{y}+\lambda u=0,
$$

где $0<\alpha<1, \lambda$ - комплексный параметр, в области $D$, ограниченной при $y>0$ кривой $\Gamma_{0}\left(x^{2}+4 y=0\right)$ с концами в точках $B(1,0)$ и $K(-1,0)$, лежащей в полуплоскости $y>0$, отрезком $A K$ оси $O X$, где $A=(0,0)$, и характеристиками $A C(x-2 \sqrt{-y}=0)$ и $C B(x+$ $2 \sqrt{-y}=1)$ уравнения (1) при $y<0$.

Пусть $D_{+}=D \cap\{y>0\}, D_{-}=D \cap\{y<0\}$.

Спектральная задача Трикоми-Неймана (задача $T N_{\lambda}$ ). Найти значения параметра $\lambda$ и соответствующие им функции $u(x, y)$, удовлетворяюшие условиям

$$
\begin{gathered}
u(x, y) \in C(\bar{D}) \cap C^{1}\left(D \cup \Gamma_{0} \cup A K\right) \cap C^{2}\left(D_{+} \cup D_{-}\right), \\
L_{\alpha} u \equiv 0, \\
u(x, y)=0, \quad(x, y) \in A K \\
u(x, y)=0, \quad(x, y) \in A C \\
\lim _{y \rightarrow 0-0}(-y)^{\alpha} u_{y}=k \lim _{y \rightarrow 0+0} y^{\alpha} u_{y}, \quad 0<x<1, \\
u_{x} d y-y u_{y} d x=0, \quad(x, y) \in \Gamma_{0}
\end{gathered}
$$

где $k=-1$ при $0<\alpha<1 / 2$ и $k=1$ при $1 / 2<\alpha<1$. 
Такой выбор значения $k$ объясняется тем, что для уравнения (1) при $\lambda=0$ доказаны теоремы существования и единственности решения задачи Трикоми-Неймана [1].

Спектральные задачи с условиями Дирихле для оператора Лаврентьева-Бицадзе были рассмотрены в работах [2]-[5]. Спектральные задачи с условиями Дирихле для уравнения (1) были изучены в статьях [6]-[8]. В [8] уравнение (1) изучалось в $D$ при $\alpha \geqslant 1$. Задача (2)-(7) при $\alpha=1 / 2$, где $k= \pm 1$, исследована в [9]. В этом случае задача $T N_{\lambda}$ заменой переменной сводится к задаче для оператора Лаврентьева-Бицадзе.

В данной работе найдены собственные значения и в явном виде построены соответствующие собственные функции задачи (2)-(7). На основании системы собственных функций решение задачи $T N$ построено в виде суммы биортогонального ряда.

2. Построение частных решений в области эллиптичности. В области $D_{+}$ перейдем к новым переменным $x=r \cos \varphi, 2 \sqrt{y}=r \sin \varphi, 0<x<1,0<\varphi<\pi$. В координатах $(r, \varphi)$ уравнение $(1)$ примет вид

$$
L_{\alpha} u=u_{r r}+\frac{1+2 \beta}{r} u_{r}+\frac{1}{r^{2}}\left(u_{\varphi \varphi}+2 \beta \operatorname{ctg} \varphi u_{\varphi}\right)+\lambda u=0, \quad \beta=\alpha-\frac{1}{2} .
$$

Разделяя в последнем уравнении переменные $u(r, \varphi)=R(r) \Phi(\varphi)$, получим

$$
\begin{gathered}
R^{\prime \prime}(r)+\frac{1+2 \beta}{r} R^{\prime}(r)+\left(\lambda-\frac{\mu^{2}}{r^{2}}\right) R(r)=0, \quad 0<r<1, \\
R(0)=0, \quad R^{\prime}(1)=0, \\
\Phi^{\prime \prime}(\varphi)+2 \beta \operatorname{ctg} \varphi \Phi^{\prime}(\varphi)+\mu^{2} \Phi(\varphi)=0, \quad 0<\varphi<\varphi_{0}=\pi, \\
|\Phi(0)|<+\infty, \quad \Phi(\pi)=0 .
\end{gathered}
$$

Известно, что решением уравнения (8) является функция Бесселя

$$
R(r)=r^{-\beta} J_{\gamma}(\sqrt{\lambda} r), \quad \gamma=\sqrt{\mu^{2}+\beta^{2}} .
$$

Подставляя (12) в краевые условия (9), имеем

$$
\operatorname{Re}(\gamma-\beta)>0, \quad \sqrt{\lambda} J_{\gamma}^{\prime}(\sqrt{\lambda})-\beta J_{\gamma}(\sqrt{\lambda})=0 .
$$

Теперь построим общее решение для уравнения (10). Для этого в (10) введем новую переменную $t=\cos ^{2}(\varphi / 2)$. Тогда оно примет вид

$$
t(1-t) \Phi^{\prime \prime}(t)+\left[\left(\frac{1}{2}+\beta\right)-(1+2 \beta) t\right] \Phi^{\prime}(t)+\mu^{2} \Phi(t)=0 .
$$

Уравнение (14) является гипергеометрическим уравнением $[10$, с. 69$]$, и поскольку $\alpha$ не является целым числом, то общее решение уравнения (10) определяется по формуле

$$
\begin{aligned}
\Phi(\varphi)= & c_{+}^{(1)} \mathrm{F}\left(\beta+\gamma, \beta-\gamma, \alpha ; \cos ^{2} \frac{\varphi}{2}\right) \\
& +c_{+}^{(2)}\left(\cos ^{2} \frac{\varphi}{2}\right)^{1-\alpha} \mathrm{F}\left(\frac{1}{2}+\gamma, \frac{1}{2}-\gamma, 2-\alpha ; \cos ^{2} \frac{\varphi}{2}\right) .
\end{aligned}
$$

Функция (15) удовлетворяет первому граничному условию из (10). Подставляя (15) во второе краевое условие из $(11)$, получим $c_{+}^{(1)}=0$.

Таким образом, частные решения уравнения $(1)$ в области $D_{+}$, удовлетворяющие краевьм условиям (7) и (4), имеют вид

$$
u=c_{+} r^{-\beta} J_{\gamma}(\sqrt{\lambda} r)\left(\cos ^{2} \frac{\varphi}{2}\right)^{1-\alpha} \mathrm{F}\left(\frac{1}{2}+\gamma, \frac{1}{2}-\gamma, 2-\alpha ; \cos ^{2} \frac{\varphi}{2}\right) \text {. }
$$


3. Построение частных решений в области гиперболичности. В уравнении (1) в области $D_{-}$сделаем замену переменных

$$
\sigma=\sqrt{x^{2}+4 y}, \quad 0<\sigma<1, \quad \theta=\frac{x+\sqrt{x^{2}+4 y}}{2 \sqrt{x^{2}+4 y}}, \quad \theta>1 .
$$

Тогда в координатах $(\sigma, \theta)$ уравнение $(1)$ примет вид

$$
\begin{gathered}
L_{\alpha} u=\theta(1-\theta) u_{\theta \theta}+\left[\left(\frac{1}{2}+\beta\right)-(1+2 \beta) \theta\right] u_{\theta} \\
+\sigma^{2} u_{\sigma \sigma}+(1+2 \beta) u_{\sigma}+\lambda \sigma^{2} u=0 .
\end{gathered}
$$

Разделяя переменные $u(\sigma, \theta)=Q(\theta) P(\sigma)$, получим

$$
\begin{gathered}
P^{\prime \prime}(\sigma)+\frac{1+2 \beta}{\sigma} P^{\prime}+\left(\lambda-\frac{\mu^{2}}{\sigma^{2}}\right) P(\sigma)=0, \quad 0<\sigma<1, \\
P(0)=0, \quad|P(1)|<+\infty, \\
\left.\theta(1-\theta) Q^{\prime \prime}(\theta)+\left[\frac{1}{2}+\beta\right)-(1+2 \beta) \theta\right] Q^{\prime}(\theta)+\mu^{2} Q(\theta)=0, \\
Q(+\infty)=0, \quad|Q(1)|<+\infty .
\end{gathered}
$$

Решением уравнения (17), удовлетворяющим условиям (18), является функция

$$
P(\sigma)=\sigma^{-\beta} J_{\gamma}(\sqrt{\lambda} \sigma), \quad \gamma=\sqrt{\mu^{2}+\beta^{2}}, \quad \operatorname{Re}(\gamma-\beta)>0 .
$$

Уравнение (19) является гипергеометрическим уравнением с аргументом $\theta>1$. Переходя к аргументу $0<1 / \theta<1$, построим его общее решение

$$
\begin{aligned}
Q(\theta)= & c_{-}^{(1)} \theta^{-(\beta+\gamma)} \mathrm{F}\left(\beta+\gamma, \frac{1}{2}+\gamma, 1+2 \gamma ; \frac{1}{\theta}\right) \\
& +c_{-}^{(2)} \theta^{-(\beta-\gamma)} \mathrm{F}\left(\beta-\gamma, \frac{1}{2}-\gamma, 1-2 \gamma ; \frac{1}{\theta}\right)
\end{aligned}
$$

Если $c_{-}^{(2)}=0$, то функция (22) удовлетворяет граничным условиям (20). Тогда решением уравнения (19), удовлетворяюшим условиям (20), будет

$$
Q_{1}(\theta)=c_{-}^{(1)} \theta^{-(\beta+\gamma)} \mathrm{F}\left(\beta+\gamma, \frac{1}{2}+\gamma, 1+2 \gamma ; \frac{1}{\theta}\right)
$$

Частньми решениями уравнения (1) в области $D_{-}$, удовлетворяющими граничному условию (5), являются функции

$$
u=c_{-}^{(1)} \sigma^{-\beta} J_{\gamma}(\sqrt{\lambda} \sigma) \theta^{-(\beta+\gamma)} \mathrm{F}\left(\beta+\gamma, \frac{1}{2}+\gamma, 1+2 \gamma ; \frac{1}{\theta}\right) .
$$


4. Построение собственных функций задачи $T N_{\lambda}$. Для нахождения собственных значений и собственных функций задачи $T N_{\lambda}$ построенную систему функций (16) и (23) подставляем в условия склеивания (2) и (6).

Из (16) и (23) вычислим

$$
\begin{aligned}
u(x, 0+0) & =\tau_{+}(x)=c_{+} x^{-\beta} J_{\gamma}(\sqrt{\lambda} x) \mathrm{F}\left(\frac{1}{2}+\gamma, \frac{1}{2}-\gamma, 2-\alpha ; 1\right), \\
u(x, 0-0) & =\tau_{-}(x)=c_{-} x^{-\beta} J_{\gamma}(\sqrt{\lambda} x) \mathrm{F}\left(\beta+\gamma, \frac{1}{2}+\gamma, 1+2 \gamma ; 1\right), \\
\lim _{y \rightarrow 0+0} y^{\alpha} u_{y}=\nu_{+}(x) & =-c_{-}(1-\alpha) x^{\alpha-3 / 2} J_{\gamma}(\sqrt{\lambda} x) \mathrm{F}(-\beta-\gamma,-\beta+\gamma, 1-\alpha ; 1), \\
\lim _{y \rightarrow 0-0}(-y)^{\alpha} u_{y}=\nu_{-}(x) & =(\beta+\gamma) c_{-}^{(1)} x^{\alpha-3 / 2} J_{\gamma}(\sqrt{\lambda} x) \mathrm{F}\left(\gamma-\beta, \frac{1}{2}+\gamma, 2 \gamma+1 ; 1\right) .
\end{aligned}
$$

Приравнивая функции

$$
\tau_{-}(x)=\tau_{+}(x), \quad 0 \leqslant x \leqslant 1, \quad \nu_{-}(x)=k \nu_{+}(x), \quad 0<x<1,
$$

получим систему

$$
\begin{aligned}
& c_{+} x^{-\beta} J_{\gamma}(\sqrt{\lambda} x) \mathrm{F}\left(\frac{1}{2}+\gamma, \frac{1}{2}-\gamma, 2-\alpha ; 1\right) \\
& =c_{-} x^{-\beta} J_{\gamma}(\sqrt{\lambda} x) \mathrm{F}\left(\beta+\gamma, \frac{1}{2}+\gamma, 1+2 \gamma ; 1\right), \\
& -c_{+}(1-\alpha) x^{\alpha-3 / 2} J_{\gamma}(\sqrt{\lambda} x) \mathrm{F}(-\beta-\gamma,-\beta+\gamma, 1-\alpha ; 1) \\
& \quad=k(\beta+\gamma) c_{-} x^{\alpha-3 / 2} J_{\gamma}(\sqrt{\lambda} x) \mathrm{F}\left(\gamma-\beta, \frac{1}{2}+\gamma, 2 \gamma+1 ; 1\right),
\end{aligned}
$$

из которой находим $\gamma$.

Рассмотрим по отдельности случаи $0<\alpha<1 / 2$ и $1 / 2<\alpha<1$. После преобразований по известным формулам $[5$, c. 111, с. 17, c. 19$]$

$$
\begin{gathered}
\mathrm{F}(a, b, c ; 1)=\frac{\Gamma(c) \Gamma(c-a-b)}{\Gamma(c-a) \Gamma(c-b)} \\
\Gamma(z) \Gamma(-z)=-\frac{\pi}{z \sin \pi z}, \quad \Gamma(2 z)=2^{2 z-1} \pi^{-\frac{1}{2}} \Gamma(z) \Gamma\left(z+\frac{1}{2}\right), \\
\Gamma(z) \Gamma(1-z)=\frac{\pi}{\sin \pi z}, \quad \Gamma\left(\frac{1}{2}+z\right) \Gamma\left(\frac{1}{2}-z\right)=\frac{\pi}{\cos \pi z}
\end{gathered}
$$

данная система сводится к тригонометрическому уравнению

$$
\cos \pi \gamma-\cos \pi(\alpha+\gamma)=0 .
$$

Отсюда с учетом того, что $\operatorname{Re}(\gamma-\beta)>0$, получим

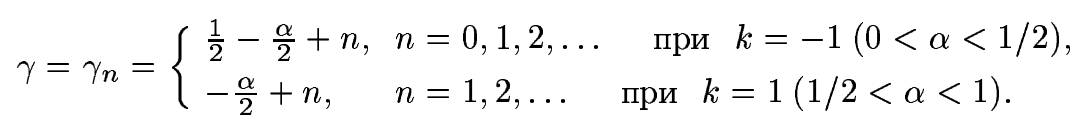


Из теории бесселевьх функций известно [11], что при $\gamma_{n}>-1$ функция $\sqrt{\lambda} J_{\gamma_{n}^{\prime}}(\sqrt{\lambda})$ $-\beta J_{\gamma_{n}}(\sqrt{\lambda})$ имеет только вещественные нули. Обозначая через $\alpha_{m}^{\gamma_{n}}-m$-й корень уравнения (13), находим собственные значения задачи $T N_{\lambda}$ :

$$
\lambda_{n, m}=\left(\alpha_{m}^{\gamma_{n}}\right)^{2}, \quad m=1,2 \ldots
$$

Тогда на основании представлений (16) и (23) с учетом значений $\lambda=\lambda_{n, m}$, получим систему соответствующих собственных функций задачи $T N_{\lambda}$ :

$$
\begin{aligned}
& u_{n, m}(x, y) \\
& =\left\{\begin{array}{l}
-k r^{-\beta} J_{\gamma_{n}}\left(\sqrt{\lambda_{n, m}} r\right)\left(\cos ^{2} \frac{\varphi}{2}\right)^{1-\alpha} \mathrm{F}\left(\frac{1}{2}+\gamma_{n}, \frac{1}{2}-\gamma_{n}, 2-\alpha ; \cos ^{2} \frac{\varphi}{2}\right),(x, y) \in D_{+}, \\
\sigma^{-\beta} J_{\gamma_{n}}\left(\sqrt{\lambda}_{n, m} \sigma\right) \theta^{-\left(\beta+\gamma_{n}\right)} \mathrm{F}\left(\beta+\gamma_{n}, \frac{1}{2}+\gamma_{n}, 1+2 \gamma_{n} ; \frac{1}{\theta}\right),
\end{array},(x, y) \in D_{-} .\right.
\end{aligned}
$$

5. Решение задачи Трикоми-Неймана. В качестве примера применения изложенных выше результатов для уравнения (1) при $\lambda=0,1 / 2<\alpha<1$ в области $D$ рассмотрим краевую задачу Трикоми-Неймана с условиями (2)-(6) и

$$
u_{x} \frac{d y}{d x}-\left.y u_{y}\right|_{\Gamma_{0}}=\hat{f}(\varphi), \quad 0 \leqslant \varphi \leqslant \pi
$$

где $\hat{f}$ - заданная достаточно гладкая функция.

Предварительно для уравнения (1) построим решение задачи Коши в области $D_{-}$.

Задача Коши. Найти в области $D_{-}$решение $u(x, y)$ уравнения $(1)$, удовлетворяющего условиям

$$
u(x, 0)=\tau(x), \quad 0 \leqslant x \leqslant 1, \quad \lim _{y \rightarrow 0-0}(-y)^{\alpha} u_{y}=\nu(x), \quad 0<x<1 .
$$

\section{Имеет место следующая}

Tеорема 1 [1, с. 111]. Если функиии $\tau(x) \in C[0,1] \wedge C^{2}(0,1), \nu(x) \in C^{2}(0,1) u$ $x^{-\beta}(1-x)^{-\beta} \nu(x) \in L(0,1)$, то существует единственное решение задачи Коии (1), (25) в области D_ и оно в характеристических координатах $(\xi, \eta)$ определяется формулой

$$
\begin{gathered}
u(x, y)=u(\xi, \eta)=k_{1}(\eta-\xi)^{1-2 \beta} \int_{\xi}^{\eta} \tau(z)(z-\xi)^{\beta-1}(\eta-z)^{\beta-1} d z \\
+k_{2} \int_{\xi}^{\eta} \nu(z)(z-\xi)^{-\beta}(\eta-z)^{-\beta} d z
\end{gathered}
$$

$2 \partial e$

$k_{1}=\frac{\Gamma(2 \beta)}{\Gamma^{2}(\beta)}, \quad k_{2}=-4^{2 \beta-1} \frac{\Gamma(2-2 \beta)}{(1-\alpha) \Gamma^{2}(1-\beta)}, \quad \xi=x-2 \sqrt{-y}, \quad \eta=x+2 \sqrt{-y}$. 
Из формулы (26) с учетом граничного условия (5) найдем

$$
\tau(x)=l \int_{0}^{x}(x-t)^{-2 \beta} \nu(t) d t, \quad l=-2 k_{2} \cos \pi \beta .
$$

В основании равенства (27) между функциями $\tau(x)$ и $\nu(x)$, принесенного из гиперболической части области $D$, задача $T N$ сведена к следующей нелокальной эллиптической задаче для уравнения (1) при $\lambda=0$ : найти в области $D_{+}$функцию $u(x, y)$, удовлетворяющую условиям (2)-(4) и (27).

Перейдем в области $D_{+}$к полярньц координатам $x=r \cos \varphi, 2 \sqrt{y}=r \sin \varphi$. Разделяя переменные $u(r, \varphi)=R(r) \Phi(\varphi)$, получим $(10),(11)$ и

$$
\begin{gathered}
R^{\prime \prime}(r)+\frac{1+2 \beta}{r} R^{\prime}(r)-\frac{\mu^{2}}{r^{2}} R(r)=0, \quad 0<r<1, \\
R(0)=0, \quad|R(1)|<\infty .
\end{gathered}
$$

Решением уравнения (28), удовлетворяющим условиям (29), является функция

$$
R(r)=r^{\gamma-\beta}, \quad \gamma=\sqrt{\mu^{2}+\beta^{2}}, \quad \operatorname{Re}(\gamma-\beta)>0 .
$$

Из п. 2 следует, что решение уравнения (10), удовлетворяющее условиям (11), имеет вид

$$
\Phi(\varphi)=c_{+}\left(\cos ^{2} \frac{\varphi}{2}\right)^{1-\alpha} \mathrm{F}\left(\frac{1}{2}+\gamma, \frac{1}{2}-\gamma, 2-\alpha ; \cos ^{2} \frac{\varphi}{2}\right) .
$$

Тогда частные решения уравнения $(1)$ в $D_{+}$, удовлетворяющие условиям $(2),(4)$, определяются по формуле

$$
u(r, \varphi)=c_{+} r^{\gamma-\beta}\left(\cos ^{2} \frac{\varphi}{2}\right)^{1-\alpha} \mathrm{F}\left(\frac{1}{2}+\gamma, \frac{1}{2}-\gamma, 2-\alpha ; \cos ^{2} \frac{\varphi}{2}\right) .
$$

Найденные функции (31) подставим в равенство (27). Предварительно вычислим

$$
\begin{aligned}
u(x, 0) & =\tau_{+}(x)=c_{+} x^{\gamma-\beta} \mathrm{F}\left(\frac{1}{2}+\gamma, \frac{1}{2}-\gamma, 2-\alpha ; 1\right) \\
\lim _{y \rightarrow 0+0} y^{\alpha} u_{y} & =\nu_{+}(x)=\lim _{\varphi \rightarrow 0}\left(\frac{r \sin \varphi}{2}\right)^{2 \alpha-1}\left(u_{r} \sin \varphi+\frac{\cos \varphi}{r} u_{\varphi}\right) \\
& =-c_{+}(1-\alpha) x^{\alpha+\gamma-3 / 2} \mathrm{~F}(-\beta-\gamma,-\beta+\gamma, 1-\alpha ; 1) .
\end{aligned}
$$

Тогда равенство (27) примет вид

$$
\begin{aligned}
& c_{+} x^{\gamma-\beta} \mathrm{F}\left(\frac{1}{2}+\gamma, \frac{1}{2}-\gamma, 2-\alpha ; 1\right) \\
& \quad=-l c_{+}(1-\alpha) \mathrm{F}(-\beta-\gamma,-\beta+\gamma, 1-\alpha ; 1) \int_{0}^{x}(x-t)^{-2 \beta} t^{\alpha+\gamma-3 / 2} d t
\end{aligned}
$$


Вычислив интеграл в правой части равенства (34), получим

$$
\mathrm{F}\left(\frac{1}{2}+\gamma, \frac{1}{2}-\gamma, 2-\alpha ; 1\right)=-l(1-\alpha) \mathrm{F}(-\beta-\gamma,-\beta+\gamma, 1-\alpha ; 1) \frac{\Gamma(2-2 \alpha) \Gamma(\beta+\gamma)}{\Gamma(1-\beta+\gamma)}
$$

Решая уравнение (35) относительно параметра $\gamma$ аналогично п. 4 , получим $\gamma=\gamma_{n}$.

Таким образом, частные решения уравнения (1), удовлетворяющие условиям (2)-(4) и (27), имеют вид

$$
u_{n}(r, \varphi)=c_{n} r^{\gamma_{n}-\beta}\left(\cos ^{2} \frac{\varphi}{2}\right)^{1-\alpha} \mathrm{F}\left(\frac{1}{2}+\gamma_{n}, \frac{1}{2}-\gamma_{n}, 2-\alpha ; \cos ^{2} \frac{\varphi}{2}\right)
$$

Перепишем это решение в другом виде. Воспользуемся формулой (6) [10, с. 144]

$$
\mathrm{P}_{\nu}^{\mu}(x)=\frac{1}{\Gamma(1-\mu)}\left(\frac{1+x}{1-x}\right)^{\frac{\mu}{2}} \mathrm{~F}\left(-\nu, \nu+1,1-\mu ; \frac{1}{2}-\frac{x}{2}\right)
$$

где $\mathrm{P}_{\nu}^{\mu}$ - модифицированная функция Лежандра. Тогда функция (39) примет вид

$$
u_{n}(r, \varphi)=f_{n} r^{\gamma_{n}-\beta}(\sin \varphi)^{1-\alpha} \mathrm{P}_{-\gamma_{n}-\frac{1}{2}}^{\alpha-1}(-\cos \varphi)
$$

где $f_{n}=c_{n} 2^{\alpha-1} \Gamma(2-\alpha)$.

Решение эллиптической задачи (2)-(4) и (27) в области $D_{+}$будем искать в виде суммы ряда

$$
u(x, y)=\sum_{n=1}^{\infty} u_{n}(r, \varphi)=\sum_{n=1}^{\infty} f_{n} r^{\gamma_{n}-\beta}(\sin \varphi)^{1-\alpha} \mathrm{P}_{-\gamma_{n}-\frac{1}{2}}^{\alpha-1}(-\cos \varphi)
$$

Предположим, что ряд (37) в $\bar{D}_{+}$сходится равномерно. Тогда, полагая в $(37) r=1$ и удовлетворяя граничному условию (24), имеем

$$
f(\varphi)=\sum_{n=1}^{\infty} f_{n}\left(\gamma_{n}-\beta\right)(\sin \varphi)^{1-\alpha} \mathrm{P}_{-\gamma_{n}-\frac{1}{2}}^{\alpha-1}(-\cos \varphi), \quad 0 \leqslant \varphi \leqslant \pi .
$$

Произведя в равенстве (38) замену $\theta=\pi-\varphi$, получим

$$
\tilde{f}(\theta)=\sum_{n=1}^{\infty} f_{n}\left(\gamma_{n}-\beta\right)(\sin \theta)^{1-\alpha} \mathrm{P}_{-\gamma_{n}-\frac{1}{2}}^{\alpha-1}(\cos \theta)
$$

где $\tilde{f}(\theta)=f(\pi-\theta)=f(\varphi)$.

На основании формулы Мейера-Дирихле [10, с. 160]

$$
\mathrm{P}_{\nu}^{\mu}(\cos \theta)=\sqrt{\frac{2}{\pi}} \frac{(\sin \theta)^{\mu}}{\Gamma\left(\frac{1}{2}-\mu\right)} \int_{0}^{\theta}(\cos v-\cos \theta)^{-\mu-\frac{1}{2}} \cos \left[\left(\nu+\frac{1}{2}\right) v\right] d v
$$


где $0 \leqslant \theta \leqslant \pi, \operatorname{Re} \mu<1 / 2$, ряд (39) примет вид

$$
\tilde{f}(\theta)=\sqrt{\frac{2}{\pi}} \frac{1}{\Gamma\left(\frac{3}{2}-\alpha\right)} \sum_{n=1}^{\infty} \int_{0}^{\theta}(\cos v-\cos \theta)^{-\beta} f_{n}\left(\gamma_{n}-\beta\right) \cos \left(v \gamma_{n}\right) d v
$$

В правой части (40) переставим порядки суммирования и интегрирования:

$$
\tilde{f}(\theta)=C_{0} \int_{0}^{\theta}(\cos v-\cos \theta)^{-\beta} \mathrm{Q}(v) d v
$$

где

$$
C_{0}=\sqrt{\frac{2}{\pi}} \frac{1}{\Gamma\left(\frac{3}{2}-\alpha\right)}, \quad \mathrm{Q}(v)=\sum_{n=1}^{\infty} f_{n}\left(\gamma_{n}-\beta\right) \cos \left(\gamma_{n} v\right)
$$

Найдем решение уравнения (41) относительно $\mathrm{Q}(v)$. Послезамены $\cos \theta=z, \cos v=t$ уравнение (41) примет вид

$$
\tilde{f}(\arccos z)=c_{0} \int_{z}^{1} \frac{\mathrm{Q}(\arccos t) d t}{\sqrt{1-t^{2}}(t-z)^{\beta}}, \quad-1 \leqslant z \leqslant 1 .
$$

Если

$$
\tilde{f}_{1}(z)=\tilde{f}(\arccos z) \in C^{1}[-1,1], \quad \tilde{f}(0)=0,
$$

то на основании известной формулы обращения интегрального уравнения Абеля получим

$$
\mathrm{Q}_{1}(z)=\mathrm{Q}(\arccos z)=-\frac{\sqrt{1-z^{2}} \sin [\pi(1-\beta)]}{\pi C_{0}} \int_{z}^{1} \frac{\tilde{f}_{1}^{\prime}(t) d t}{(t-z)^{1-\beta}} .
$$

Тогда

$$
\begin{aligned}
& \sum_{n=1}^{\infty} f_{n}\left(\gamma_{n}-\beta\right) \cos \left(\gamma_{n} \arccos z\right)=-\frac{\Gamma(1-\beta) \sin \pi \beta}{\sqrt{2 \pi}} \sqrt{1-z^{2}} \\
& \times \int_{z}^{1} \tilde{f}_{1}^{\prime}(t)(t-z)^{\beta-1} d t=\mathrm{Q}_{1}(z) .
\end{aligned}
$$

Ряд (43) является биортогональным разложением функции $\mathrm{Q}_{1}(z)$ по системе косинусов $\left\{\cos \gamma_{n} \theta\right\}_{n=1}^{\infty}$. В силу результатов [12] ряд (43) сходится к функции $\mathrm{Q}_{1}(z)$ в пространстве $L_{p}(0 ; \pi)$ при $p<(1-\alpha)^{-1}$, при этом коэффициенты ряда вычисляются по формулам

$$
f_{n}=\frac{\sin \pi \beta}{\left(\gamma_{n}-\beta\right) \sqrt{2 \pi}} \Gamma(1-\beta) \int_{0}^{\pi} h_{n}(\theta) \sin \theta d \theta \int_{0}^{\theta} \frac{\tilde{f}^{\prime}(v) d v}{(\cos v-\cos \theta)^{1-\beta}}
$$

где

$$
h_{n}(\theta)=\frac{2}{\pi(2 \cos \theta / 2)^{\alpha}}\left[\sum_{m=0}^{\infty} C_{\alpha}^{m} \cos (n-m) \theta-\frac{C_{\alpha}^{k}}{2}\right] .
$$

В силу теоремы $3.6[13$, с. 65$]$, если $p>1 /(\alpha-1 / 2)$, то $\mathrm{Q}_{1}(z) \in C^{\alpha-1 / 2-1 / p}[-1,1]$. Отсюда в силу результатов работы [12] следует равномерная сходимость ряда (43). 
Условия (42) будут вьполнены, если $f(\varphi) \in C^{1}[0, \pi]$, функция $f(\varphi)$ в малой окрестности точек $\varphi=0$ и $\varphi=\pi$ дважды непрерьвно дифференцируема, $f(0)=f^{\prime}(0)=$ $f(\pi)=f^{\prime}(\pi)=0$.

При этом биортогональная система $\left|h_{n}(\theta)\right|$ равномерно ограничена по $k$; следовательно, коэффициенты $f_{n}$ равномерно ограничены по $n$, поэтому ряд (37) при $r \leqslant 1$ сходится равномерно. Также равномерно при $r<1$ сходятся ряды, полученные путем дифференцирования ряда (37) по переменным $r$ и $\varphi$ любое число раз. Поэтому ряд (37) является решением задачи Трикоми-Неймана в области $D_{+}$.

Для построения решения задачи Трикоми-Неймана в области $D_{-}$найдем след производной $y^{\alpha} u_{y}$ от решения (36) на отрезке $A B$. Имеем

$$
\lim _{y \rightarrow 0+0} y^{\alpha} u_{y}=\lim _{\varphi \rightarrow 0}\left(\frac{r \sin \varphi}{2}\right)^{2 \alpha-1}\left[\sin \varphi u_{r}+\frac{\cos \varphi}{r} u_{\varphi}\right] .
$$

Предварительно найдем производные $u_{r}, u_{\varphi}$ :

$$
\begin{aligned}
u_{r}= & \sum_{n=1}^{\infty}\left(\gamma_{n}-\beta\right) f_{n}(\sin \varphi)^{1-\alpha} r^{\gamma-\beta-1} \mathrm{P}_{-\gamma_{n}-1 / 2}^{\alpha-1}(-\cos \varphi) \\
u_{\varphi}= & \sum_{n=1}^{\infty} f_{n}(1-\alpha)(\sin \varphi)^{-\alpha} \cos \varphi r^{\gamma-\beta} \mathrm{P}_{-\gamma_{n}-1 / 2}^{\alpha-1}(-\cos \varphi) \\
& +\sum_{n=1}^{\infty} f_{n}(\sin \varphi)^{1-\alpha} r^{\gamma_{n}-\beta} \frac{d}{d \varphi} \mathrm{P}_{-\gamma_{n}-1 / 2}^{\alpha-1}(-\cos \varphi) .
\end{aligned}
$$

Используя формулы [10, с. 162, с. 163]

$$
\begin{gathered}
\left(1-x^{2}\right) \frac{d \mathrm{P}_{\nu}^{\mu}(x)}{d x}=(\nu+1) x \mathrm{P}_{\nu}^{\mu}(x)-(\nu-\mu+1) \mathrm{P}_{\nu+1}^{\mu}(x), \\
(\nu-\mu+1) \mathrm{P}_{\nu+1}^{\mu}(x)-(\nu+\mu+1) x \mathrm{P}_{\nu}^{\mu}(x)=\sqrt{1-x^{2}} \mathrm{P}_{\nu}^{\mu+1}(x),
\end{gathered}
$$

имеем

$$
\begin{aligned}
& \frac{d}{d \varphi} \mathrm{P}_{-\gamma_{n}-1 / 2}^{\alpha-1}(-\cos \varphi) \\
& \quad=\left(-\gamma_{n}+\frac{1}{2}\right)(-\cos \varphi) \mathrm{P}_{-\gamma_{n}-1 / 2}^{\alpha-1}(-\cos \varphi)-\left(-\gamma_{n}-\alpha+\frac{3}{2}\right) \mathrm{P}_{-\gamma_{n}+1 / 2}^{\alpha-1}(-\cos \varphi)
\end{aligned}
$$

Тогда

$$
\begin{aligned}
u_{\varphi}= & \sum_{n=1}^{\infty} f_{n}(\sin \varphi)^{1-\alpha} r^{\gamma_{n}-\beta}\left[\left(-\gamma_{n}+\alpha-\frac{1}{2}\right)(-\cos \varphi) \mathrm{P}_{-\gamma_{n}-1 / 2}^{\alpha-1}(-\cos \varphi)\right. \\
& \left.-\left(-\gamma_{n}-\alpha+\frac{3}{2}\right) \mathrm{P}_{-\gamma_{n}+1 / 2}^{\alpha-1}(-\cos \varphi)\right] \\
= & -\sum_{n=1}^{\infty} f_{n} r^{\gamma_{n}-\beta}(\sin \varphi)^{1-\alpha} \mathrm{P}_{-\gamma_{n}-1 / 2}^{\alpha}(-\cos \varphi)
\end{aligned}
$$


Теперь вычислим $\tau(x)$ и $\nu(x)$. Поскольку при $\varphi \rightarrow 0$ справедливы асимптотические представления [10, с. 165]

$$
\begin{aligned}
\mathrm{P}_{-\gamma_{n}-\frac{1}{2}}^{\alpha-1}(-\cos \varphi) & \sim \frac{\Gamma(1-\alpha)\left(\sin \frac{\varphi}{2}\right)^{\alpha-1}}{\Gamma\left(\frac{3}{2}-\alpha+\gamma_{n}\right) \Gamma\left(\frac{3}{2}-\alpha-\gamma_{n}\right)}, \\
\mathrm{P}_{-\gamma_{n}-\frac{1}{2}}^{\alpha}(-\cos \varphi) & \sim \frac{1}{\pi} \cos \pi \gamma_{n} \Gamma(\alpha)\left(\sin \frac{\varphi}{2}\right)^{-\alpha},
\end{aligned}
$$

то

$$
\begin{aligned}
\lim _{\varphi \rightarrow 0}(\sin \varphi)^{2 \alpha} u_{r} & =0, \quad \lim _{\varphi \rightarrow 0}(\sin \varphi)^{2 \alpha-1} \cos \varphi u_{\varphi}=-\frac{1}{\pi} \Gamma(\alpha) \sum_{n=1}^{\infty} f_{n} r^{\gamma_{n}-\beta} \cos \pi \gamma_{n}, \\
\nu(x) & =\lim _{y \rightarrow 0+0} y^{\alpha} u_{y}=-\frac{1}{\pi} \Gamma(\alpha) \sum_{n=1}^{\infty} f_{n} x^{\gamma_{n}+\alpha-3 / 2} \cos \pi \gamma_{n} \\
\tau(x) & =u(x, 0)=\sum_{n=1}^{\infty} f_{n} x^{\gamma_{n}-\beta} \frac{\Gamma(1-\alpha)}{\Gamma\left(1-\beta+\gamma_{n}\right) \Gamma\left(1-\beta-\gamma_{n}\right)} .
\end{aligned}
$$

Теперь подставляя (45) и (46) в формулу (26), получим

$$
\begin{aligned}
u(\xi, \eta)= & \frac{\Gamma(2 \beta)}{\Gamma^{2}(\beta)}(\eta-\xi)^{1-2 \beta} \int_{\xi}^{\eta} \sum_{n=1}^{\infty} f_{n} \Gamma(1-\alpha) \frac{z^{\gamma_{n}-\beta}(z-\xi)^{\beta-1}(\eta-z)^{\beta-1} d z}{\Gamma\left(1-\beta+\gamma_{n}\right) \Gamma\left(1-\beta-\gamma_{n}\right)} \\
& +4^{2 \beta-1} \frac{\Gamma(\alpha) \Gamma(2-2 \beta)}{(1-\alpha) \pi \Gamma^{2}(1-\beta)} \\
& \times \int_{\xi}^{\eta} \sum_{n=1}^{\infty} f_{n} \cos \pi \gamma_{n} z^{\gamma_{n}+\alpha-3 / 2}(z-\xi)^{-\beta}(\eta-z)^{-\beta} d z .
\end{aligned}
$$

Поменяв порядки суммирования и интегрирования, имеем

$$
\begin{aligned}
u(\xi, \eta)= & \frac{\Gamma(2 \beta)}{\Gamma^{2}(\beta)}(\eta-\xi)^{1-2 \beta} \sum_{n=1}^{\infty} f_{n} \frac{\Gamma(1-\alpha)}{\Gamma\left(1-\beta+\gamma_{n}\right) \Gamma\left(1-\beta-\gamma_{n}\right)} \\
& \times \int_{\xi}^{\eta} z^{\gamma_{n}-\beta}(z-\xi)^{\beta-1}(\eta-z)^{\beta-1} d z \\
& +4^{2 \beta-1} \frac{\Gamma(\alpha) \Gamma(2-2 \beta)}{(1-\alpha) \pi \Gamma^{2}(1-\beta)} \\
& \times \sum_{n=1}^{\infty} f_{n} \cos \pi \gamma_{n} \int_{\xi}^{\eta} z^{\gamma_{n}+\beta-1}(z-\xi)^{-\beta}(\eta-z)^{-\beta} d z .
\end{aligned}
$$

Воспользуемся следующей формулой $[14$, с. 301$]$ :

$$
\begin{aligned}
& \int_{a}^{b}(x-a)^{\lambda-1}(b-x)^{\beta-1}(c x-d)^{\delta} d x \\
& \quad=(b-a)^{\lambda+\beta-1}(a c+d)^{\delta} \mathrm{B}(\lambda, \beta) \mathrm{F}\left(\lambda,-\delta, \lambda+\beta ; \frac{c(a-b)}{a c+d}\right),
\end{aligned}
$$


где $\operatorname{Re} \lambda>0, \operatorname{Re} \beta>0$, для вычисления интегралов из правой части (47). Тогда

$$
\begin{gathered}
\int_{\xi}^{\eta} z^{\gamma_{n}-\beta}(z-\xi)^{\beta-1}(\eta-z)^{\beta-1} d z=(\eta-\xi)^{2 \beta-1} \xi^{\gamma-\beta} \mathrm{B}(\beta, \beta) \mathrm{F}\left(\beta, \beta-\gamma, 2 \beta ; \frac{\xi-\eta}{\xi}\right) \\
\int_{\xi}^{\eta} z^{\gamma_{n}+\alpha-\frac{3}{2}}(z-\xi)^{-\beta}(\eta-z)^{-\beta} d z \\
\quad=(\eta-\xi)^{1-2 \beta} \xi^{\gamma+\alpha-\frac{3}{2}} \mathrm{~B}(1-\beta, 1-\beta) \mathrm{F}\left(1-\beta, \frac{3}{2}-\alpha-\gamma, 2-2 \beta ; \frac{\xi-\eta}{\xi}\right)
\end{gathered}
$$

На основании формулы $[10$, с. 113$]$

$$
\mathrm{F}(a, b, c ; z)=(1-z)^{b} \mathrm{~F}\left(c-a, b, c ; \frac{z}{z-1}\right)
$$

имеем

$$
\begin{gathered}
\mathrm{F}\left(\beta, \beta-\gamma, 2 \beta ; \frac{\xi-\eta}{\xi}\right)=\left(\frac{\eta}{\xi}\right)^{\gamma-\beta} \mathrm{F}\left(\beta, \beta-\gamma, 2 \beta ; \frac{\eta-\xi}{\eta}\right) \\
\mathrm{F}\left(1-\beta, 1-\beta-\gamma, 2-2 \beta ; \frac{\xi-\eta}{\xi}\right) \\
=\left(\frac{\eta}{\xi}\right)^{\gamma+\beta-1} \mathrm{~F}\left(1-\beta, 1-\beta-\gamma, 2-2 \beta ; \frac{\eta-\xi}{\eta}\right) .
\end{gathered}
$$

Тогда решение (47) примет вид

$$
\begin{aligned}
u(\xi, \eta)= & \Gamma(1-\alpha) \sum_{n=1}^{\infty} f_{n} \eta^{\gamma_{n}-\beta} \frac{\mathrm{F}\left(\beta, \beta-\gamma_{n}, 2 \beta ; \frac{\eta-\xi}{\eta}\right)}{\Gamma\left(1-\beta+\gamma_{n}\right) \Gamma\left(1-\beta-\gamma_{n}\right)} \\
& +\frac{4^{2 \beta-1} \Gamma(\alpha)}{(1-\alpha) \pi}(\eta-\xi)^{1-2 \beta} \\
& \times \sum_{n=1}^{\infty} f_{n} \cos \pi \gamma_{n} \eta^{\gamma_{n}+\beta-1} \mathrm{~F}\left(1-\beta, 1-\beta-\gamma_{n}, 2-2 \beta ; \frac{\eta-\xi}{\eta}\right)
\end{aligned}
$$

Отметим, что решение (48) удовлетворяет граничному условию (5). Следовательно, функция (48) является решением задачи Трикоми-Неймана в области $D_{-}$.

Итак, доказано следующее утверждение.

Tеорема 2. Ecли $f(\varphi) \in C^{1}[0, \pi], f(0)=f^{\prime}(0)=f(\pi)=f^{\prime}(\pi)=0$, и в малой окрестности точек $\varphi=0 u \varphi=\pi$ дважды непрерывно дифференцируема, то существует единственное решение задачи Трикоми-Неймана в области $D u$ оно определяется формулами (37) $и$ (48), где коэффициенты $f_{n}$ определяются по формулам (44).

Аналогично строятся решение задачи $T N$ для уравнения (1) при $\lambda=0,0<\alpha<1 / 2$ и решение задачи $T N$ для уравнения $(1)$ при $\lambda \neq \lambda_{n, m}$. 


\section{СПИСОК ЦИТИРОВАННОЙ ЛИТЕРАТУРЫ}

[1] Смирнов М. М. Уравнения смешанного типа. М., 1985.

[2] Пономарев С. М. Спектральная теория основной краевой задачи для уравнения смешанного типа Лаврентьева-Бицадзе. Автореферат дисс. ... д-ра физ.-матем. наук. М.: МГУ, 1981.

[3] Моисеев Е.И. Уравнения смешанного типа со спектральным параметром. М.: МГУ, 1998.

[4] Моисеев Е.И. Решение задачи Трикоми в специальных областях // Дифференц. уравнения. 1990. Т. 26. №1. С. 93-103.

[5] Сабитов К. Б., Тихомиров В. В. О построении собственных значений и функций одной газодинамической задачи Франкля // Матем. моделирование. 1990. Т. 2. № 10. С. 100-109.

[6] Мамедов Я. Н. О некоторых задачах на собственные значения для уравнения смешанного типа // Дифференц. уравнения. 1990. Т. 26. №1. С. 163-168.

[7] Моисеев Е.И. О представлении решения задачи Трикоми в виде биортогонального ряда // Дифференц. уравнения. 1991. Т. 27. № 7. С. 1229-1237.

[8] Сабитов К. Б., Вагапов В.З. О построении частных решений вырождающихся уравнений смешанного типа // Комплексный анализ, дифференц. уравнения и смежные вопросы. Тр. Международ. науч. конф. Уфа, 1996. С. 99-106.

[9] Бибакова С. Л. Задача на собственные значения для уравнения смешанного типа // Тр. Всеросс. науч. конф. "Физика конденсированного состояния". Т. 1. Стерлитамак, 1997. C. $14-18$.

[10] Бейтмен Г., Эрдейи А. Высшие трансцендентные функции. М.: Наука, 1973.

[11] Ватсон Г. Н. Теория бесселевых функций. Т. 1. М., 1949.

[12] Моисеев Е. И. О базисности одной системы синусов // Дифференц. уравнения. 1987. Т. 23. № 1. C. $177-179$.

[13] Самко С.Г., Килбас А. А., Маричев О.И. Интегралы и производные дробного порядка и некоторые их приложения. Минск: Наука и техника, 1987.

[14] Прудников А.П., Брычков Ю.А., Маричев О. И. Интегралы и ряды. М.: Наука, 1981.

Стерлитамакский филиал АН Республики Башкортостан

Поступило

Стерлитамакский государственный педагогический институт

14.11.2001 